\title{
An Updates on Techniques of Microsurgical Vasectomy Reversal- A Mini Review
}

\section{Dr. Kulvinder Kochar Kaur, MD ${ }^{1 *}$, Dr. Gautam Allahbadia, MD (Obstt \& Gynae), D. N. B ${ }^{2}$ Dr. Mandeep Singh, MD, DM. (Std) (Neurology)}

${ }^{1}$ Scientific Director, Dr Kulvinder Kaur Centre for Human Reproduction, Punjab, India.

${ }^{2}$ Scientific Director, Ex-Rotunda-A Centre for Human reproduction, Mumbai, India.

${ }^{3}$ Consultant Neurologist, Swami Satyanand Hospital, Punjab, India.

*kulvinder.dr@gmail.com

*Corresponding Author: Dr. Kulvinder Kochar Kaur, MD, Scientific Director, Dr Kulvinder Kaur Centre for Human Reproduction, Punjab, India.

\begin{abstract}
Following sterilization by vasectomy, men my need future fertility, either due to the loss of a child or a new marriage. Following vasectomy reversal there have been a lot of advances in the field to improve the patency and pregnancy rates, although in the modern era of assisted reproductive technology they are usually not counseled with the ease of sperm retrieval and IVF/ICSI. Here we carried out a systematic analysis using the MeSH terms on the search engine Pubmed, like "vasectomy reversal", "vasovasostomy'(VV),' "vasoepididymostomy"(VE), "optimizing success", "different sutures" to be used, "most modern techniques" like robotic assisted VV or VE, Video microscopy, analysis of vasal fluid". We found a total of 1200 articles, out of which we selected 75 articles for this review. No meta-analysis was carried out. Further emphasis has been laid on the training of new urologists to learn this complicated technique so that natural conception can be sought for. Further techniques of some complicated cases are described.
\end{abstract}

Keywords: VV; VE; Vasectomy reversal; IVF/ICSI/Vasal fluid

\section{INTRODUCTION}

Vasectomy reversal is the recommended method of reversing sterilization in men desiring fertility subsequently. With marked improvements in techniques in the last 4 decades, high patency rates and favourable pregnancy outcomes following reconstruction can be expected. Though there are other treatment options that are available for these patients with post vasectomy obstructive azoospermia (OA) meaning different kinds of sperm retrieval techniques, vasectomy reversal is the only method which allows natural conception. Further Vasectomy reversal has also been found to be more cost effective in contrast to sperm retrieval to be used for IVF (in vitro fertilization)/ICSI (Intracytoplasmic injection). Still the success of Vasectomy reversal is based on good clinical judgement, efficient microsurgical skills and proper selection of subjects. The aim of this review is to highlight the treatment approach for vasectomy reversal in length, based on the recent evidence. The advances made recently in surgical technique and how they contribute to this surgery are to be critically analyzed. Since they are technically problematic stress is put on role of microsurgical training, that includes the current knowledge which are improving the education in this field.

\section{Evaluation to be done Preoperatively}

Preoperative examination of men with suspected OA starts with a proper history taking and thorough examination physically. Main idea of this is to find the cause of azoospermia whether obstructive (OA) or non obstructive azoospermia (NOA). Specially on examination of the spermatic cord, a heating blanket or hot pack is placed with the idea of warming the scrotum, thus relaxing the scrotal dartos muscle. Still no sign or symptom is $100 \%$ sensitive or specific and this early examination can be further increased in case of borderline cases. 
An Updates on Techniques of Microsurgical Vasectomy Reversal- A Mini Review

In case of a clear causative factor identification, laboratory examination is usually not needed, like an early history of vasectomy. But in absence of vasectomy, or if patient had difficulty in getting a pregnancy, prior to sterilization, it is better to get an FSH and morning time testosterone (T) level. This discrimination of $\mathrm{OA}$ vs NOA was carried out by Schoor et al. [1]. A length of testis of a minimum $4.6 \mathrm{~cm}$, in combination with an $\mathrm{FSH}$ level $<7.6 \mathrm{mIU} / \mathrm{ml}$, identifies subjects having OA in $96 \%$ of the patients. With this the preoperative testicular biopsy has got practically removed for diagnosing. Though useful, the Schoor criteria will wrongly diagnose obstruction in roughly 1in 20 men. Subjects with NOA, having an appropriate testicular size, and a FSH value $<7.6 \mathrm{mIU} / \mathrm{ml}$, usually have some form of maturation arrest on histology. Hung et al. [2] examined a cohort of 600 men who presented with NOA where services were provided by a single unit. $4.3 \%$ of the subjects had a testicular length $>4.6 \mathrm{~cm}$ and a normal FSH level, with maturation arrest on biopsy. Even Tsai et al. [3] on examination of men presenting with maturation arrest, observed both testis volume and a FSH level below the threshold in $38 \%$ of the cohort they studied. Because of this any reproductive urologist who asks the patient for reconstructive surgery needs to know the limitations of the basic laboratory tests, since operative plan may change dramatically in the possible misdiagnosis. Serum antisperm antibodies assessment is useful for confirming spermatogenesis; A high positive assay confirms the diagnosis in case of OA [4].

Mostly imaging studies don't give much information, but for suspected ejaculatory duct obstruction, where a transrectal ultrasound shows dilated seminal vesicles and/or ejaculatory ducts or midline cysts might be diagnostic. Ultrasonography (USG) doesn't provide any additional data over physical examination in case of vasal gaps, presence or absence of a sperm granuloma, or testicular size (if an orchid meter is utilized). Only way USG might be helpful is in getting the diameter of caput epidymis, which will differentiate OA/NOA, but not for a specific patient having maturation arrest -all these are the most important points regarding preoperative examination [5]. Further fullness of the epidydimis might not be present in men having $\mathrm{OA}$, where obstruction is at the level of rete testis, which may be present in $15 \%$ of men with OA [6].

On confirming obstruction, further patient counseling should include anticipated reconstructive outcomes.
In the work done by the Vaso-vasostomy Study Group, best chances of success get obtained when the interval of obstruction was under 3 years (patency rate 97\%, pregnancy rate $76 \%$ [7]. With increasing duration of vasal encounters the outcomes kept on worsening, with 15 plus years of obstruction leading to patency and pregnancy rates of $71 \%$ and $30 \%$ respectively. Once obstructive interval rises chances of secondary epididymal increase occurs and thus the patient requires both vasoepididymostomy (VE). Technically VE is more difficult, with greater likelihood of early and late failures, besides reduced pregnancy rates, inspite of patency getting demonstrated [8, 9]. Fuch's etal. showed a $50 \%$ increase in the probability of needing a VE for every 3 year period following vasectomy in one of the biggest published series. Performance rate of VE falls once the level of obstruction reaches the efferent ducts, secondary to inadequate epididymal exposure following spermatogenesis [11, 12]. Since the prognosis is based largely on if bilateral VE will be needed, many groups have chalked out extra preoperative predictors for this setting. McCammack et al. [13] tried to use magnetic resonance imaging for laying down the prognosis of requirement of IVF in OA. They had a small cohort of 10 subjects with known OA, a 19. 4\% increase in epididymal T1intensity predicted the need for VE in $90 \%$ of subjects. Follow up studies are required to confirm these findings which are consisting of large cohorts. Once McCammack's finding get confirmed, magnetic resonance imaging (MRI) might prove a useful tool for prediction of epididymal obstruction and the subsequent requirement of VE instead of vasovasostomy (VV). Patients need to understand prior to the surgery that bilateral VE is needed as patency and pregnancy rates are typically reduced in such circumstances [7].

In terms of importance this impact of interval since obstruction has not been clear in contemporary series $[14,15]$. Still most of literature implicates that the interval of obstruction remains one of the most important factor regarding preoperative counseling [16]. Other important factor in preoperative counseling is whether sperm granuloma is present. It is considered that a sperm granuloma provides a passage to relieve vasal pressure, thus decreasing the risk of secondary epidymal obstruction by "blowout" [17]. In contrast to obstruction interval, sperm granuloma was considered favourable in older series, having more studies recently showing little or no predictive 
value $[7,14,15,18]$. Boorjian et al. presented a large experience of a single surgeon, finding more chance of statistical significance $(\mathrm{p}=0.07)$ regarding difference in patency rates following a vasectomy reversal $(95 \%$ vis a vis $78 \%$ for subjects without a palpable sperm granuloma). Despite that some examine for sperm granuloma, since a granuloma might be pointing to a greater -quality intravasal fluid during the reconstruction and hence imply a greater chance that one can perform a $\mathrm{VV}$ procedure that is lesser tough $[7,18]$.

Other aspects on history/general physical examination (GPE), include history of previous vasectomy reversal, big vas al gaps, if any iatrogenic vasal injury is reflected by another procedure carried out earlier like herniorrhaphy. A lot of small series presented success following previous failed reversal, found moderately decreased success rates. The Vasovasostomy Study Group presented a series of 199 subjects in which a repeat procedure was done, and found a patency rate in $75 \%$ of subjects, with a pregnancy rate of $43 \%$ [7]. A cohort done following that consisted of 41 patient who had at least 1 previous attempt at vasal reconstruction reported by Hernandez etal. [19] Found a patency rate and pregnancy rate of $79 \%$ and $31 \%$ respectively. They showed that $39 \%$ of their subjects needed bilateral VE that gives importance of preoperative knowledge. Once large vasal gaps or iatrogenic injury to vas is present, it points to the inability of vas to get a tension -free anastamosis. Which should be discussed with the patient prior to the intervention [20]. Although such settings are rare, surgical approach varies markedly from the traditional VV and VE and may even need specialized equipment [21]. No long term outcomes are available regarding these reconstructions which are full of complexity. Patient attempting these need to be aware of lack of information as regards patency and pregnancy rate anticipated.

\section{Female Factor}

Clinical characteristics of the female partner must be considered while making the decision on treatment plan for OA, Since these factors will affect prognosis as significantly as the males preoperative evaluation. Currentreproductiveurologisthas differentprocedures to offer men following vasectomy that range from definitive reconstruction to the different kinds of sperm retrieval techniques. In some cases like tubal factor, the couples are forced to have IVF and hence sperm retrieval is the best option. However except for these cases where IVF is unavoidable, utilization of vasectomy reversal has decreased markedly in the post-ICSI era. Although many cases of increased use of ART, exist, one major cause is that women delay the family building [23]. It is clear that with increasing maternal age there is an inverse relation to chances of natural conception and ART outcomes. Further, men wanting fertility following vasectomy tend to be older and might have partners who are of high age as well.

Following vasectomy reversal main factor which predicts pregnancy, besides re-establishment of patency, is the age of the female partner. According to Megheli et al. [14], who described a cohort of 334 subjects who underwent reconstruction and found that female age was the only independent predictor of pregnancy, that outperformed obstructive interval and sperm granuloma status. Just like that on examination of 173 men undergoing vasectomy reversal for obstructive intervals of at least 15years Fuchs et al. [8] found that pregnancy rates of $64 \%$ and $32 \%$ according to partners, having $<30$ year age vis a vis $36-$ 40 years respectively. Thus the overall pregnancy rate was $43 \%$, that was similar to the contemporary IVF/ ICSI results. Since it can sometimes take $>6 \mathrm{mths}$ for sperms to return to the ejaculate, and hence successful pregnancy might even take longer, some consider that vasectomy reversal is not the choice of treatment for couples that have partner of advanced maternal age $[9,24,25]$. Stratification was attempted in a better way by Kapadia et al. [26] as per womans age. They analyzed 136 men who chose to undergo vasectomy reversal even if their partners were 35 years or older and compared the result they obtained with the ART results that had been documented. They found that live birth rates were comparable between per cycle IVF data and vasectomy reversal. For subjects with partners over 40 years, the vasectomy reversal group got a birth rate of $15.4 \%$, while one cycle of ART gave a birth rate of $14.2 \%$, when selecting for couples who did not have any separate female infertility factors.

Still whether vasectomy reversal is proper when the woman is $>35 y$ years remains unanswered since Kapadia et al. [26] just compared per cycle outcome while many vasectomy reversal couples in the long run will undergo multiple cycles. Still all subjects seeking vasectomy reversal it is worth offering that vasectomy reversal is more cost effective than ART. Lee et al. [27] utilized a constructive and analytical 
An Updates on Techniques of Microsurgical Vasectomy Reversal- A Mini Review

model for comparison of vasectomy reversal with sperm retrieval along with IVF/ICSI, which included indirect costs [27]. In terms of dollars the calculated cost in 2005 for vasectomy reversal, microsurgical epididymal sperm with IVF/ICSI, and testicular sperm aspiration with IVF/ICSI were, 25321\$, 58528\$ and $61,977 \%$ respectively. This was similar to the cost factor analysis by Heidenreich etal. [28], that further adds to much less morbidity with complications if vasectomy reversal was opted for.

Finally history of proven fertility in the partner in the couples who present is another important preoperative factor. In the earlier studies that contained small cohorts found high pregnancy rates when the male partner was seeking pregnancy with the same female partner prior to vasectomy. The study carried out by the Vasovasostomy Study Group, subjects that underwent reconstruction secondary to death of a child, got a pregnancy rate of $76 \%(n=21)$, while in subjects having a new partner got a pregnancy rate of $50 \%(n=612)[7]$. On the same lines subjects with the same partner, Kolettis etal. [29] and Chan etal. [30] Obtained pregnancy rates of $60 \%$ and $86 \%$ respectively. Ostrowsky et al. [31], conducted a comparison of 258 couples where partner was same with the rest of vasectomy reversal cohort. A pregnancy rate of $83 \%$ were obtained when vasectomy reversal was done in a couple with proven fertility in contrast to $60 \%$ in the other cohort giving an odds ratio of $2.0(\mathrm{P}<0.01)$. For reasons not very clear prognosis when partner is same is markedly greater, which should be emphasized in preoperative counseling.

Thus the criteria combining both male and his wife will update regarding the prognosis for any attempt carried out at vasectomy reversal. Thus the reproductive urologist has to make a decision along with the male partner weighing al pros and cons. Though vasectomy reversal is the gold standard and is most cost effective for all patient reporting, sperm retrieval might prove the most effective choice in certain situations [32]

\section{Vasectomy Reversal: Techniques}

The techniques of vasectomy reversal got optimized in 1980s and further in 1990s with marked work of the Vasovasostomy Study Group [7, 33]. The basics of either VV or VE depend on a good quality anastamosis which is a tension free, mucosa to mucosa connection that maintains adequate blood supply, The anastamosis has to be water tight for maximizing the chances of patency. In view of crossover recombination, sperm will produce an inflammatory response when presented outside of the immune privileged space of the testis [34]. If leakage of sperm occurs from a reconstruction side will result in a granuloma development, that might distract the anastamosis, causing failure [35, 36]. Further to achieve a high quality anastamosis in the male reproductive tract, where the vasal lumen is as small as $0.3 \mathrm{~mm}$ and epididymal tubules as small as $0.15 \mathrm{~mm}$, proper visualization with an operating microsope is pivotal[37,]. Though reasonable patency can be obtained using loupes and prolene stent, expected results are still lesser than the traditional microsurgical reconstruction that maintains the option of doing $\mathrm{V} E$ if required [37].

Both types of vasectomy reversal, VV o r VE, can be done using a high bilateral scrotal incision. Some workers have retried a modified technique utilizing a small midline incision via no scalpel vasectomy maneuvers [38]. Although patency and pain outcomes were favourable, the limited surgical exposure VE and can be rather hindering for large vasal gaps or significant granulomas. The group of Hayden prefers the traditional high scrotal exposure since it is generally well tolerated and gives marked flexibility for an unexpected different reconstruction. Further high bilateral scrotal incisions can be used even in awake procedures, although most surgeons prefer anaesthesia should VE gets indicated [36, 39].

Decision making following an intraoperative approach

Irrespective of the incision opted for, the vasectomy reversal begins with the idea of notification of the vssal gap and resection of nonviable vas deferens with the help of a slotted nerve clamps provide comparable outcomes [40]. Distal patency must be ensured to confirm practicality of reconstruction, that might be obtained through a saline or water soluble contrast vasogram [35]. If obstruction of the abdominal vas remnant might occur due to inadequate resection of the vasectomy site, or due to unrecognized iatrogenic injuries from procedures that occurred after sterilization (e.g herniorrhaphy) [41]. In the rare scenario of inguinal and abdominal obstruction (usually from previous hernia repair) crossed VV might be of use in case the contralateral testis has a patent epidydimis (as confirmed by the presence of sperm in testicular and vasal fluid, while the ipsilateral epidydimis is blocked [42]. 
An Updates on Techniques of Microsurgical Vasectomy Reversal- A Mini Review

For particular cases, assessing the vasal end might be the first decision point for the surgeon. The vasal ends should be patent, having proper blood supply as assessed by bleeding from the final mucosal vessels. What is of importance is that mucosal vessels will usually bleed following a short delay, while adventitial vessels will bleed briskly following transection. Usually the testicular remnant will determine better perfusion. The surgeon should be able to see 4 layers: mucosa, two layers of muscle (subtle), and the adventitia. If 2 muscular layers are not seen, it might indicate residual scar from the vasectomy. More resection for optimizing the vasal ends must be weighed against the criterion of a larger vasal gap, a choice that can be helped by experience via adequate microsurgical training.

On obtaining homeostasis for the testicular vasal remnant with the use of a low-power bipolar cautery, intravasal fluid can be examined following that without obscuring contamination with red blood cells. Macro and microscopic appearance of the vasal efflux tells the surgeon whether secondary epididymal obstruction has occurred, thus obliterating VE. One can anticipate, finding of motile sperm within vas deferens rules out the possibility of epididymal obstruction, with post VV patency rates nearing $100 \%$ for some series [43]. The presence of non motile but intact sperms also predicts high patency rates. For this latter case, the Vasovasostomy Study Group showed a patency rate of $91 \%$ to $96 \%$ [7]. On visualizing only sperm parts (i. e sperm heads), the Vasovasostomy Study Group showed a patency rate of $75 \%$. Subsequent smaller studies found similar results, on the basis of which many providers carried out a VE in such a setting [44]. Scovell et al. [45] Carried out a contemporary metaanalysis that included 1239 subjects across 6 studies, re-examined the role of intravasal fluid assessment that neared statistical significance during vasectomy reversal. Observation of sperm parts or sperms with short tails positively correlated with pregnancy, rendering the recommendations for VV in such a case. Subsequently a retrospective review was carried out in 902 subjects, where finding of only sperm parts in intravasal fluid assessment neared statistical significance for worse post reversal pregnancy outcomes $(p=0.05)$ [46]. Still most providers now go on with VV when only sperm parts are found, since the expected patency rates are greater than for VE. Presence of intravasal azoospermia might indicate
VE; yet the surgeon must interpret the macroscopic findings of the vasal efflux to make sure the operative plan that is going to be.

Overall, the vasal efflux can be differentiated on the basis of its colour, consistency and volume. If there is "toothpaste" like fluid, that is a part of the vassal epithelium, and in the absence of sperm, it warrants VE $[47,48]$. But plenty of clear fluid following transaction of vas deferens generally indicates a patent epididymis, even in the absenceof sperm $[7,48]$. Problem exists if there is intravasal azoospermia, with a low efflux volume, or if the colour/consistency is different from clear and thin. In this context Hayden et al. advocate opening the tunica vaginalis [49]. A transition point of dilated of dilated to nondilated epididymal tubules is a clear sign of secondary obstruction. Though a transition point is specific, it is not very sensitive, and its absence does not rule out epididymal blockade [50]. Other strategies are barbotage of the testicular vassal remnant in hopes of discovering sperm or waiting a short period of time, (i.e. while the contralateral side is examined), that sometimes might result in return of very few sperms. Because of this it is always important to evaluate both sides prior to finalizing which technique is to be used.

An important factor that can complicate the assessment of intravasal fluid is the commonly used $\mathrm{T}$ replacement therapy (or less common, anabolic steroids). Exogenous androgens suppress the release of gonadotropins centrally. Although systemic T levels might reach therapeutic levels, spermatogenesis needs intratesticular concentrations to be higher in quantity [51] In the absence of intratesticular $\mathrm{T}$ synthesis that is stimulated by $\mathrm{LH}$, there will be impaired sperm production because of inadequacy of exogenous hormone replacement therapy. So much so that azoospermia was induced in $95 \%$ of subjects in a study with the use of high dose $\mathrm{T}$ undecanoate [52]. Thus the subjects presenting for vasectomy reversal with recent use of androgens might present with intravasal azoospermia because of poor synthesis rather than due to epidydimal blockade. Recently Coward et al. [53] treated 6 patient with clomiphene and /or HCG prior to vasectomy reversal. They showed expected rates of intravasal sperm during surgery. Bigger studies are required for confirming these early results especially with the testis recovery is complex following exogenous androgen exposure [54]. 


\section{An Updates on Techniques of Microsurgical Vasectomy Reversal- A Mini Review}

\section{Vasovasostomy}

Following assessment of vasogram and intravasal fluid if $\mathrm{VV}$ is needed one must assess the resulting vasal gap by freeing both vasal remnants to make sure that there is a tension free anastamosis. One must take care that vasal vasculature is preserved, for preventing failure because of ischemia. Better mobility and length can be obtained usually from the testicular remnant, especially when the convoluted vas is freed. In case of large vassal gaps, more advanced procedures might be required like inverting the test is to add to the supplied length [42]. On bringing both ends of the vas together without tension, microspike approximator use helps in exposure along with fixation [42]. Importance has to be given to see that vas deferens does not twist while setting up the reconstruction. Hayden et al. utilize the microdot technique as well for preplanning placement of every suture, which is very helpful if luminal discrepancy exists [43] (fig 1).
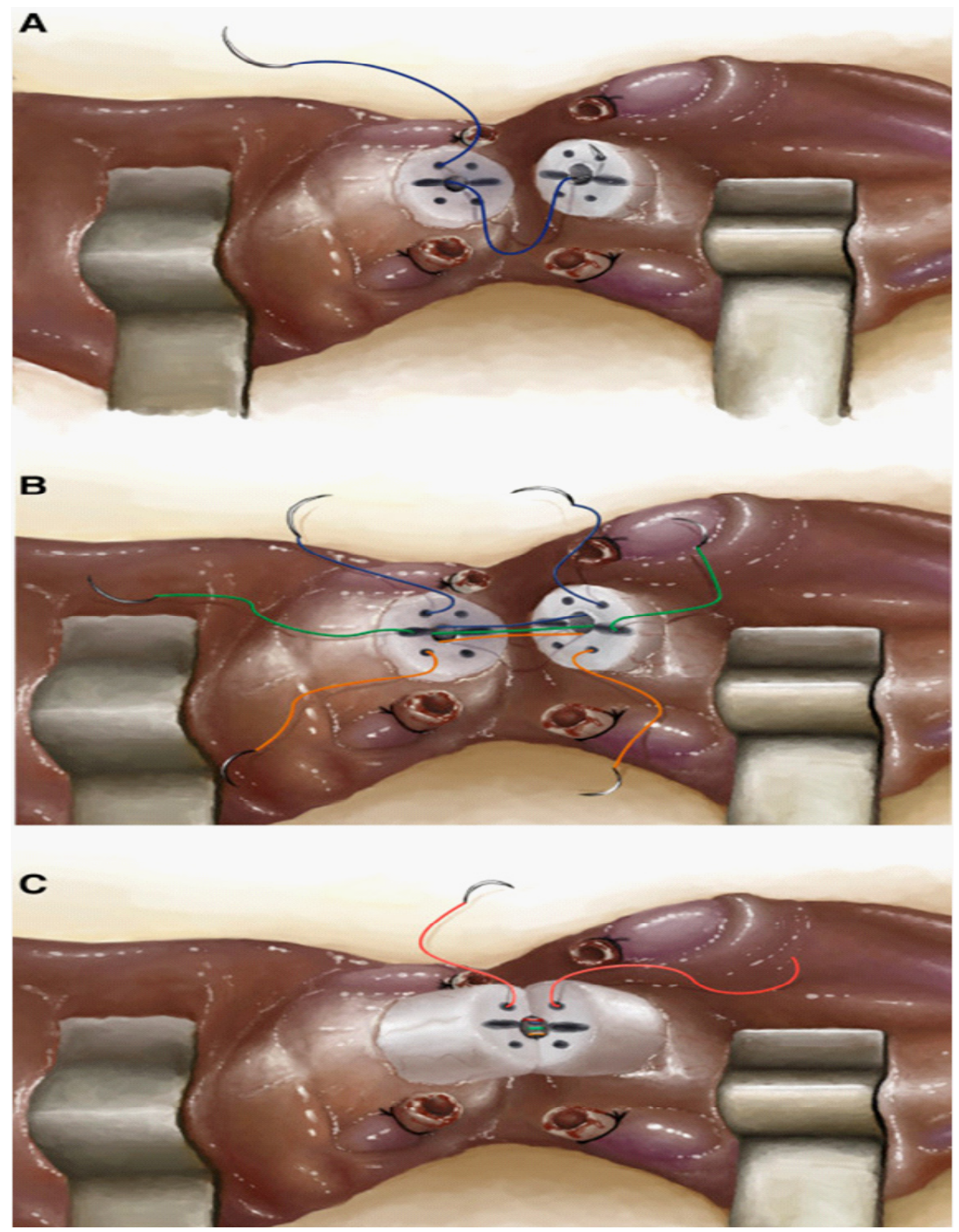

Fig 1. Courtesy ref no 49. (A) Setup and placement of the mucosal sutures for VV. (B) Note that the initial three sutures must form a "three-string guitar" before tying, a heuristic that helps ensure no sutures inadvertently crossed each other. (C) Flipping of the microspike approximator facilitates the remaining mucosal sutures. With this exposure the initial three ties should be visible and checked for inadvertent back-walling during initial suture placement. 
The Vasovasostomy Study Group did not find any statistical difference in patency or pregnancy outcomes for two layer and single layer VVs [7]. Following that a meta-analysis confirmed these results where 6,633 patients were involved [16]. Possibly, a high quality single layer anastamosis might prevent extravasation and thus reduce the accompanying risk of granuloma. But Hayden et al. continue to use the two layer technique, since that it has been associated with greater patency rate that has been published $[43,49]$. For highlighting the mucosa indigo carmine can be used prior to placing the sutures. A total of six 10-0 sutures get used for approximating the mucosa, with additional six 9-0 sutures placed to approximate
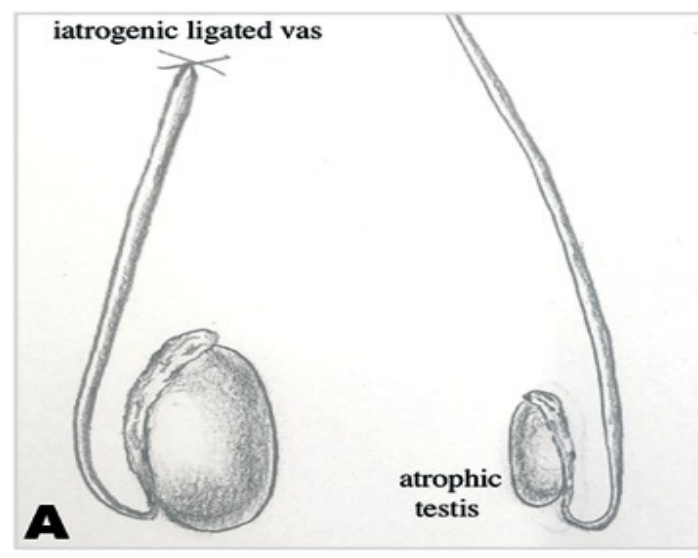

the muscularis. Adventitia is further brought together with at least six 9-0 sutures. Ultimately the adventitia is brought together with 7-0 polydioxanone sutures, that give additional tensile strength to the repair. Animal studies have indicated noninferiority of nylon sutures for the 10-0 and 9-0 layers [55]. To prevent back walling double-armed sutures are a must since all sutures are put inside out [49]. In some challenging cases cross microsurgical atypical vasovas ostomy in scrotum of atypical OA [56]. (FIG 2, 3, 4). Similarly crossover transseptal vasovas ostomy has been considered as an alternative for very selected cases of iatrogenic injury to vas deferens. [57] (fig 5, 6).

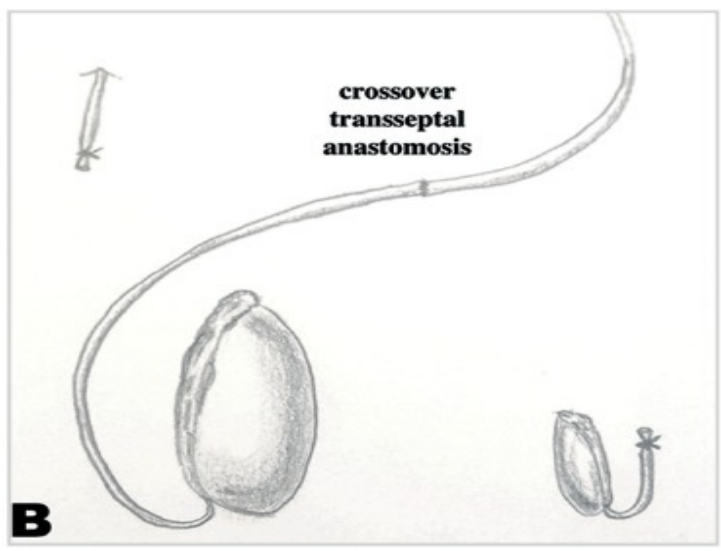

Fig 2. Courtesy ref no 49.-Steps for successful LIVE. See text ("Vasoepididymostomy" section) for a written description.

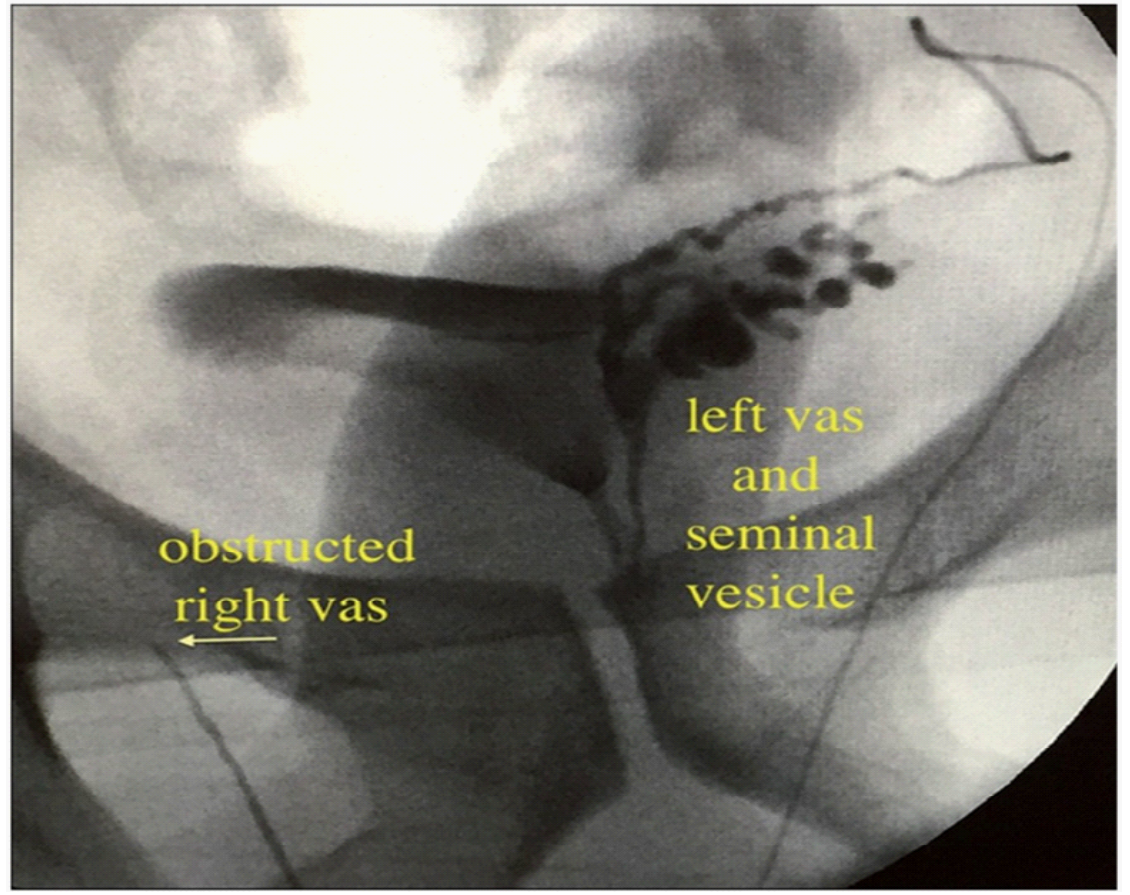

Fig 3. Courtesy ref no. 56Intraoperative deferentography demonstrating obstructed right vas deferens and normal left vas and seminal vesicle. 
An Updates on Techniques of Microsurgical Vasectomy Reversal- A Mini Review

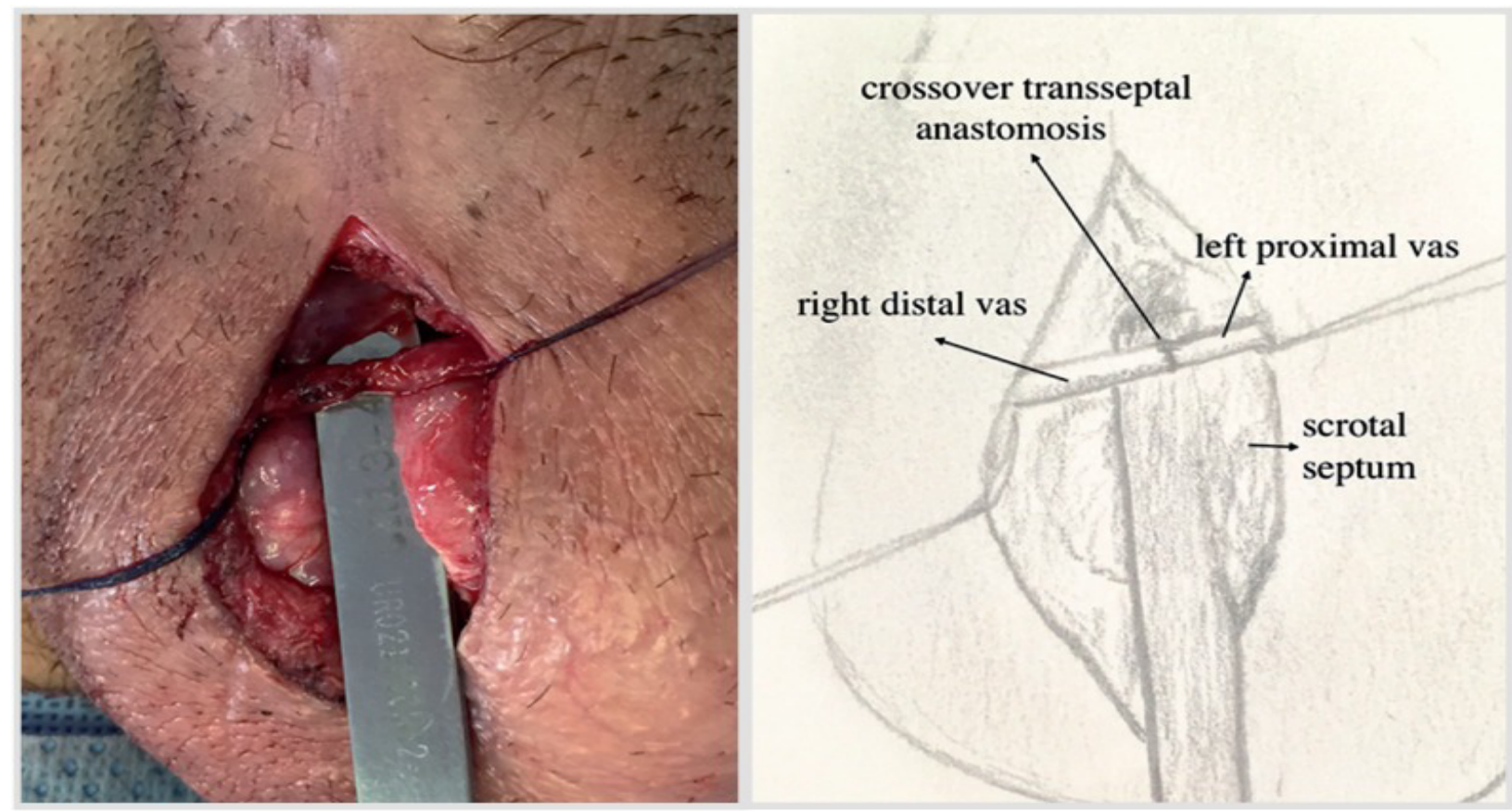

Fig 4. Courtesy ref no 56.A) Schematic view of iatrogenic injury to right vas deferens with hypotrophic left testis. B) Schematic view of surgical procedure for microsurgical crossover transseptal vasovasostom

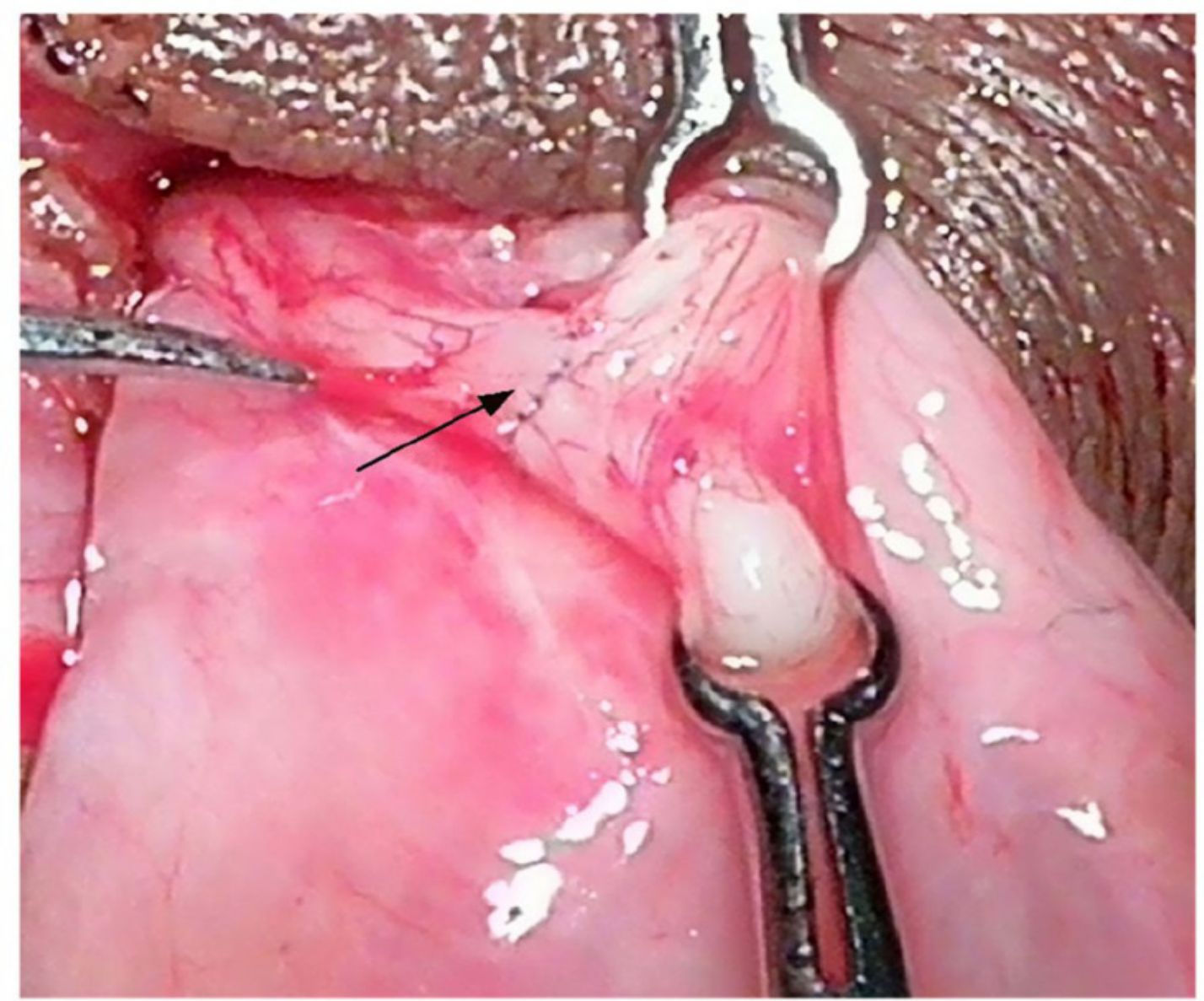

Fig 5. Courtesy ref no 56. Final aspect of the microsurgical crossover transseptal vasovasostomy. 
An Updates on Techniques of Microsurgical Vasectomy Reversal- A Mini Review

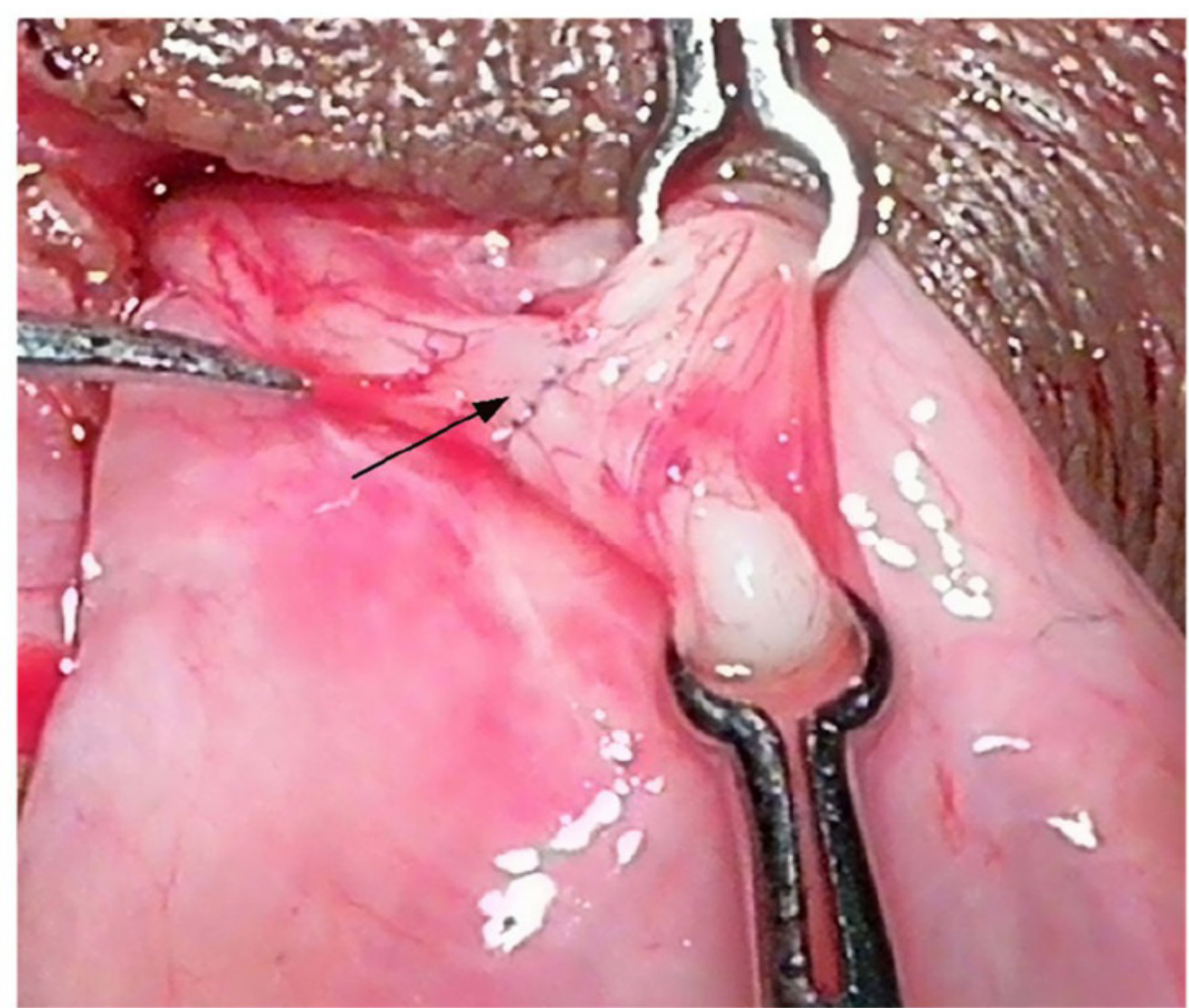

Fig 6. Courtesy ref no 57-Cross vasovasostomy in the scrotum The arrow refers to the anastomosis site

A

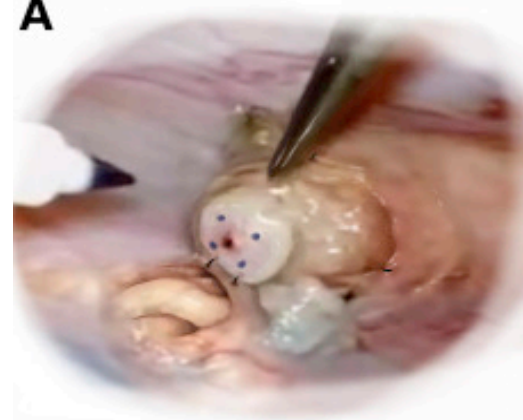

B

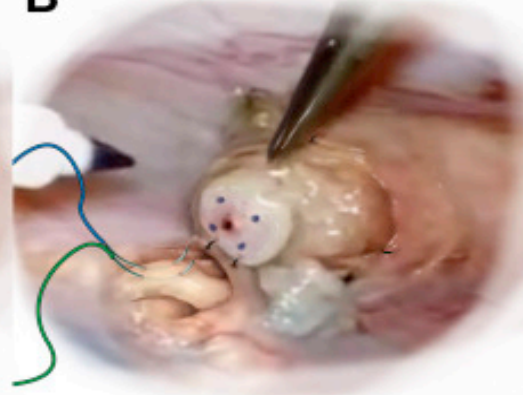

E

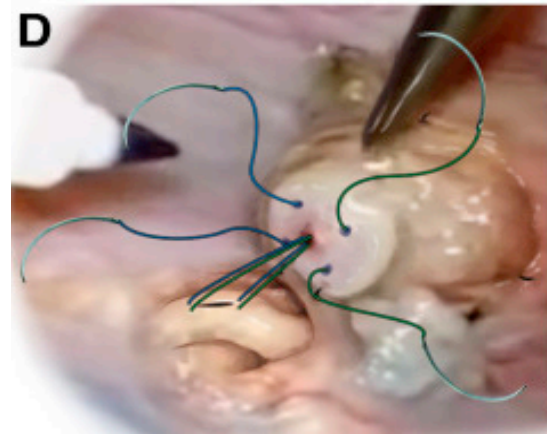

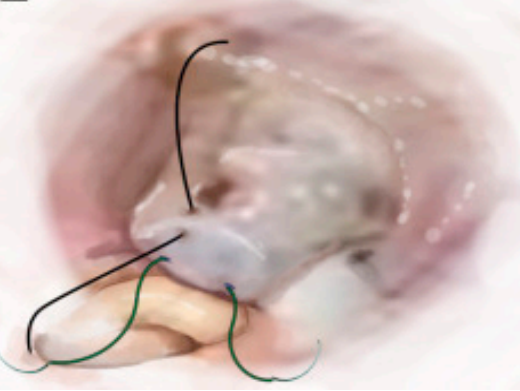

C

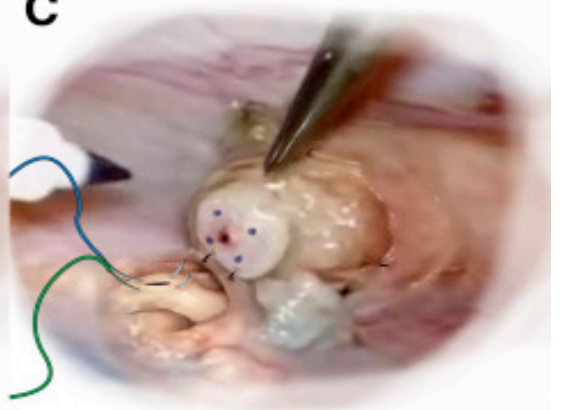

$\mathbf{F}$

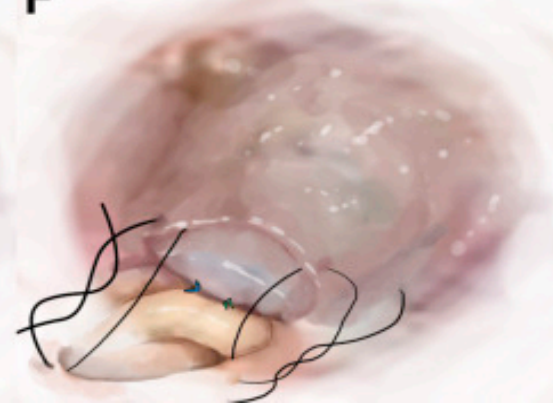

Fig 7. Courtesy ref no-Cross vasovasostomy in the scrotum The arrow refers to the anastomosis site 
An Updates on Techniques of Microsurgical Vasectomy Reversal- A Mini Review

\section{Vasoepididymostomy}

VE is much more difficult as compared to VV in view of the size and fragility of epidymal tubules. Still the technique needs to be mastered by any surgeon attempting vasectomy reversal as the presence or absence of epididymal obstruction can be judged only intraoperatively. [58]Just like VV, a sufficient length of the abdominal vasal remnant is essential to be obtained so that tension freeanastamosis can be performed. Since the abdominal vas gives less ability to free more length, occasionally testis might have to be pexed superiorly for avoiding undue stress on the resulting reconconstruction [42]. Following that a small window is made in the tunica vaginalis, and the abdominal remnant is drawn through to facilitate the reversal.

How to select the epididymal tubule is guided by the tubule size, its orientation, and the natural lay of the vas deferens. Sinc e patency outcomes tend to drop as more epididymal length is excluded, the initial tubule which is selected should be as distal as is possible, but above any transition points which might be present [12]. Small window of epididymal tunic is then excised by gently raising the tissue with jeweler forceps and transected with the help of microscissors. Inadvertent entry into an epididymal tubule means to repeat attempt upstream within the epididymis. Then the vas is anchored to the edge of the tunical window with 9-0 nylon sutures (fig7A). This might be helped with the use of approximator clamp, since any tension might tear these delicate tissues.

There are multiple techniques for VE, although the Longitudinal Intusussecption vasoepididymostomy (LIVE) procedure has been found to be better in animal studies and now is the one considered as the standard of care [59]. The selected epididymal tubule is $1^{\text {st }}$ linked with indigo carmine for helping watch the lumen once a tubulotomy is made. Two 10-0 double armed nylon sutures are then put longitudinally along the tubule in a parallel fashion. It is crucial that the needles are left in situ for preventing deflation of the epidermal tubule, that will make the tubulotomy overly traumatic otherwise (fig 7B and 7C). Further placing the needles in the beginning, would allow enough space for making a tubulotomy. Then with the help of ophthalmic knife the tubules between the needle then incises the tubules between the needles. Then the effluxed fluid needs to be examined for the presence/ absence of sperms. Once no sperms are found, this site needs to be given up and look for a new tubule upstream. If sperms are present, the already placed double armed sutures are then placed through the mucosa of the vassal end (fig 7D). Just as in VV, Hayden et al. mark the vas with microdots for simplifying the sututure passage. Following that 9-0 stay suture for bringing the vas lumen down into the tubulotomy by securing the vasal adventititia to the tunical window, directly opposite to the initial anchoring stitch (fig 7E). Prior to tying the 10-0 sutures, careful tension should be applied till all snack has been removed(as indicated by subsequent movement of the opposite side of the stitch ). This step also makes sure that proper identification of both legs of the same suture, which need to be tied together at that time. Finally step of VE is to secure the rest of the vas circumference to the tunical window window with multiple interrupted 9-0 Nylons (fig 7F). Spacing of sutures will ensure a watertight seal.

Little modifications of this LIVE technique have been described in the different publications. Various trials have examined, the use of single armed 10-0 sutures [60]. The patency which results following use of single armed 10-0sutures tend to be lesser than with the use of traditional double -arm ed sutures. To reduce back walling Hayden et al. use only double-armed sutures. Zhang et al 61]. Made another modification, a vessel sparing VE, that was meant for pts with a long history of varicocoele repair. Larger series that corroborat this have not been published. Still the improved success of LIVE technique and its simplicity decrease the chances of errors.

\section{New Tools}

Advances in surgical tools have given more chances for improving microsurgical reconstruction. The biggeste. $\mathrm{g}$ is robotic surgery, since it offers high resolution, three dimensional magnification, with tremor -reduced fine manipulation by different surgical graspers. Earliest publications regarding possibility and effectivity of robotic -assisted VV on fresh human vas deferens specimens was given by Kuang et al. [62]. Different animal studies were subsequently carried out for assessing patency outcomes. The only randomized controlled trial was conducted by Schiff et al. [63] where they compared robotic VV and VE with the operating microscope done in a rat model. Following 9 weeks of recovery these rats were killed and examined 
for patency and granuloma formation. The time taken for surgery for $\mathrm{VV}$ were considerably lower in the robotic group, while no difference in operating time was noted in VE. No statistical difference in patency rates were observed between the 2 instruments. Sperm granuloma formation was higher significantly in the microscopic VV, with no difference in rates of VV.

So far various small studies have reported noninferior outcomes of robotic vasectomy reversal in humans [64]. Yet the uptake of robotic vasectomy reversal has been infrequent, probably due to cost factor and the traditionally trained microsurgeons being more comfortable with the operating microscope. Though cost -efficacy of robotic -assisted vasectomy reversal is debatable, it may be excellent in difficult cases where intracorporeal vasal reconstruction has to be done [65]. Larger series are needed to corroborate this approach and verify the durability of patency following extended follow up.

Video microsurgery is another technology, which is coming up and might be used for vasectomy reversal. Here instead of the operating microscope, a high resolution camera which transmits to monitors that are capable of producing three-dimensional images with accompanying eye wear gets utilized [66]. Till now, the technology for obtaining the needed resolution for facilitating safe visualization using this procedure was limited. Recently, both Olympus and Zeiss are manufacturing products which surpass the imaging limits needed for surgery. Video microscopy has promise for a longer working focal length, wider field of view, along with minimal surgeon strain due to posture [67]. More studies are needed for confirming the projected performance of video microscopes vis a vis the traditional operating microscope.

\section{Post Operative Manipulation}

In many Western countries vasectomy reversal is conducted as a day procedure. Convalescence of patient is similar to that for minor scrotal surgeries, that are well tolerated. Complications are haematoma, and infrequently infection. These patients are followed up by most reproductive urologists with serial semen analysis. Farber et al. [9] conducted a meta-analysis, analyzing the kinetics and later failure rates were delineated for the pooled data of 24 studies. Sperm return to the ejaculate happened between a mean range of $1.7-4.3 \mathrm{mths}$ for $\mathrm{VV}$ and upto $6.6 \mathrm{mths}$ for
VE. Late failures, by definition is a return of seminal azoospermia, that occurred in upto $12 \%$ and $50 \%$ for VV and VE, respectively. In view of high rates of the anastamosis getting shut down it is better to counsel the patient for sperm cryofreezing once patency has been confirmed.

The reference values for semen analysis following vasectomy reversal had not been clear till now. A cohort of 139 subjects were examined following vasectomy reversal by Majzoub et al. [68]. They found that men who were able to achieve a pregnancy had much lower semen parameters in contrast to WHO referral values [69]. A sperm concentration of $<5 \times 10^{6} / \mathrm{ml}$, motility $<10 \%$ and morphology $<1 \%$ a pregnancy rate observed were $15 \%, 21.3 \%$, and $14 \%$ respectively [68]. Though the studies cohort was small, there initial data give an early means of interpreting the postreversal semen analysis beyond the WHO, $5^{\text {th }}$ edition criteria and the binary outcome of patency [69].

\section{Training of the Surgeons}

Since vasectomy reversal is a procedure which requires adequate attention it needs dedicated microsurgical training to make sure optimum results are achieved for the patients. Tissue handling technique, needle control of 10-0 sutures, intraoperative assessment of vasal stumps, tension, and epididymal tubule selection can only be acquired via experience rather than from published literature. With male infertility fellowships quiet common, this field will continue to further superspecialize and thus there will be condensation into centres of excellence. In other urologic subspecialities, learning curve s for common surgeries are being defined for allowing quality control of the practitioners. For this the best example comes from oncology field, where minimal case load to carry out a robotic-assisted prostatectomy is being described [70].

Need for standardization and quality control of reproductive urologists has been noticed for a longtime. Wood et al. [71] surveyed both urologists along with general surgeons who were practicing vasectomy reversal to assess the volume and outcomes. Those who submitted high volumes ( $>15 /$ year) obtained a patency rate of $87 \%$ in contrast to those practitioners having lesser volume ( $<6 /$ year), having a patency rate of 56\%. Nagler et al. [72] observed similar results, i. e. patency rates of $89 \%$ and $53 \%$ respectively, for those who did and did not practice the skills in a 
An Updates on Techniques of Microsurgical Vasectomy Reversal- A Mini Review

microsurgical lab. A rat model is used accurately to simulate both the VV and VE techniques in Hayden etal. Centre [73]. Just like that the Toronto group also gave promising results from their training curriculum [74].

Description of learning curve for the traditional microsurgical vasectomy reversal is a goal that has not been reached till now. Kavoussi et al [75]. Described the learning curve for the transition of a skilled microsurgeon to robotic-assisted vasectomy reversal for a single high volume reproductive urologist. It was found patency rates remained consistent and within acceptable limits over time, but operative times significantly reduced following the $1^{\text {st }} 50$ cases. Thus a continuous work is required to mature reproductive urology for standardizing and optimizing training of future guidelines.

\section{ConClusions}

Techniques of microsurgical vasectomy reversal keep on evolving continuously for improving outcomes for males who want fertility following elective sterilization. Success begins with proper pinpointing of proper candidates for this surgery preoperatively. Need for assessing both partners is a must and very important for postoperative $\mathrm{OA}$, and proper preop examination and counseling can help in choosing the proper treatment plan. Even after choosing vasectomy reversal the surgeon might face various intraoperative decisions making on the basis of remaining healthy anatomy along with examination of intravasal fluid. In view of the great amount of work by the Vasovasostomy Study Group, most treatment algorithm is known, despite some occasional questions regarding if only sperm parts are found. High rates of patency with favourable pregnancy outcomes can be ensured to the pts. For continuous delivery of quality care, reproductive urologists need to standardize fellowship education, and pass it on to the next generation of subspecialists.

\section{REFERENCES}

[1] Schoor RA, Elhanbly RS, Niederberger CS, Ross LS. The role of testicular biopsy in the modern management of male infertility. J Urol $2002 ; 167$ : 197-200.

[2] Hung AJ, King P, Schlegel PN. Uniform testicular Maturation arrest: a unique subset of men with obstructive azoospermia. J Urol 2007; 178: 608-12.
[3] Tsai MC, Cheng YS, Lin TY, Yang WH, Lin YM. Clinical characteristics and reproductive outcomes in infertile men with testicular early and late maturation arrest. Urology, 2012; 80: 826-32.

[4] Lee R, Goldstein M, Ullery BW, Ehrlich I, Soares $M$ Razaano RA et al. Value of serum sperm antibodiesindiagnosingobstructiveazoospermia. J Urol 2 009; 181: 264-9.

[5] Pezella A, Barbonetti A, D’Andreas S, Neconzione S, Micillo A, Di Gregorio A, et al. Ultrasonographic caput pididymis diameter is reduced in non obstructive azoospermia compared with normozoospermia but is not predictor for successful sperm retrieval after TESE. Hum Reprod 2014; 29: 1368-74.

[6] Miyaoka R, Esteves SC, Predictive factors for sperm retrieval and sperm injection outcomes in obstructive azoospermia: do etiology, retrieval techniques and gamete source play a role? Clinics (Saulo Paulo)2013; 68 (Suppl1): 111-9.

[7] Belker AM, Thomas AJ Jr, Fuchs EF, Konnak JW, Sharlip ID. Results from1469 microsurgical vasectomy reversal by the Vaso Vasostomy Study Group. J Urol 1991; 145: 505-11.

[8] Fuchs EF, Burt RS. Vasectomy reversal performed 15years or more after vasectomy: correlation of pregnancy outcome with partner age and with pregnancy results of invitro fertilization and intracytoplasmic sperminjection. Fertil Steril 2002; 77: 51 6-9.

[9] Farber EF, Flannigan R, Li P, Li PS, Goldstein M. The kinetic of spermreturn and late failure following vasovasostomy and vasoepididymostomy: a systematic review. J Urol 2019; 201: 241-50.

[10] Fuchs ME, Anderson RE, Ostrowski Ka, Brant WO, Fuchs EF, Preoperative risk factors associated withneed for vasoepididymostomy at the time of vasectomy reversal. Andrology 2016; 4: 160-2.

[11] Moore HD, Hartman TD, Pryor JP, Development of the oocyte-penetration capacity of spermatozoa in the human epididymis. Int J Androl 1983; 6: 310-8.

[12] Pasqualotto FF, Agarwal A, Srivastava M, Nelson DR, Thomas AJ Jr. Fertility outcome after rapeat vasoepididymostomy. J Urol1999; 162: 1626-8. 
An Updates on Techniques of Microsurgical Vasectomy Reversal- A Mini Review

[13] McCammack KC, Aganovic L, Hsieh TC, Guo Y, Welch CS, Gamst AC, et al. MRI of the epididymis: can the outcome of vasectomy reversal be predicted preoperatively? AJR Am J Roentgenol 2014; 203: 91-8.

[14] Magheli A, Rais-Bahrami S, Kempkensteffen C, Weiske WH, Miller K, Hinz S. Impact of obstructive interval and sperm granuloma on patency and pregnancy outcome after vasectomy reversal. Int J Androl2010; 33: 730-5.

[15] Boorjian S, Lipkin M, Goldstein M, The impact of obstructive interval and sperm granuloma on outcome of vasectomy reversal. J Urol; 2004; 171: 304-6.

[16] Herrel LA, Goodman M, Goldstein M, Hsiao W. Outcomes of microsurgical vasovasostomy for vasectomy reversal: a meta-analysis and systematic review. Urology 2015; 85: 819-25.

[17] Johnson AL, Howards SS. Intratubular hydrostatic pressure in testis and epididymis before and after vasectomy. Am J Pathol1975; 228: 556-64.

[18] Belker AM, Konnak JW, Sharlip ID, Thomas AJJr. Intraoperative observation duringvasovasostomy in 334 patients. J Urol 1983; 129: 524-7.

[19] Hernandez J, Sabanegh ES, Repeat vasectomy reversal after initial failure: overall results and predictors for success. J Urol 1999; 161: 1153-6.

[20] Mathews GJ, Mc Gee KE, Goldstein M, Microsurgical reconstruction following failed vasectomy reversal. J Urol 1997; 157: 844-6.

[21] Barazani Y, Kaouk J, Sabanegh ES Jr. Robotic intraabdomial vasectomy reversal: a new approach to a different problem. Can Urol Assoc J 2014; 8: E439-41.

[22] Pastuszak AW, Sigalos JT, Lipshultz LI. The role of the urologist in the era of in vitro-fertilizationintracytoplasmic sperm injection. Urology 2017; 103: 19-26.

[23] Mathews TJ, Hamilton BE. Mean age of mother, 1970-2000. Natl Vital Rep2002; 51: 1-13.

[24] Valerie U, De Brucker S, De Brucker M, Vloeberghs V, Drakopoulos P, Santos-Ribeiro S, et al. Pregnancy after vasectomy: surgical reversal or assisted reproduction? Hum Reprod 2018; 33: 1218-27.
[25] Practice Committeee of the American Society of Reproductive Medicine in Collaboration with the Society for male Reproduction and Urology. The agreement of infertility due to obstructive azoospermia. Fertil Steril 2008; 90 (Suppl): S121-4.

[26] Kapadia AA, Anthony M, Martinez-Acevedo A, Fuchs EF, Hedges K, Ostrowsk KA. Reconsidering vasectomy reversal over assisted reproduction in older couples. Fertil Steril 2018; 109: 1020-4.

[27] Lee R, LeePS, Goldstein M, Tanrikut C, Schattman $G$, Schlegel PN. A decision analysis of treatments for obstructive azoospermia. Hum Reprod 2008; 23: 2043-9.

[28] Handenreich A, Altman P, Engelmann UH. Microsurgical vasovasostomy versus microsurgical epidydimal sperm aspiration/ testicular extraction of sperms combined with intracytoplasmic sperm injection: A cost benefit analysis. Eur Urol 2000; 37: 609-14.

[29] Kolettis PN, Woo L, Sandow JI. Outcomes of vasectomy reversal performed for men with the same female partners. Urology 2003; 61: 1221-3.

[30] Chan PT, Goldstein M. Superior outcomes of microsurgical vasectomy reversal in men with same female partners. Fertil Steril 2004; 81: 1371-4.

[31] Ostrowski KA, Polachwich AS, Kent J, Conlin MJ, Hedges JC, Fucks EF. Higher outcomes of vasectomy reversal in men with the same female partner as before vasectomy. J Urol 2015; 193: 245-7.

[32] American Society of Reproductive Medicine: Sperm retrieval for obstructive azoospermia. Fertil Steril 2008; 90 (5 Suppl): S213-8.

[33] Silber S. Microscopic vasectomy reversal. Fertil Steril 1977; 28: 1191-202.

[34] Mruk DD, Cheng CY. The mammalian-blood -testis barrier: its biology and regulation. Endocr Rev 2015; 36: 564-91.

[35] Chan PT. The evolution and refinement of vasoepididymostomy techniques. Asian J Androl 2013; 15: 49-55.

[36] Patel AP, Smith RP. Vasectomy reversal: A clinical update. Asian J Androl 2016; 18: 365-71. 
An Updates on Techniques of Microsurgical Vasectomy Reversal- A Mini Review

[37] Lee HY. A 20year experience with vasovasostomy. J Urol 1986; 136: 413-5.

[38] Jarvi K, Kwon T, Park S, Cheon SH, Moon KH. Loupe -assisted vaso-vasostomy using a prolene stent: a simpler Vasectomy reversal technique. World J Mens Health 2017; 35: 115-9.

[39] Alom M, Ziegelmann M, Savage I, Meist T, Kohler TS, Trost L. Office based andrology and male infertility procedure-a cost effective alternative. Transl Androl Urol 2017; 6: 761-72.

[40] Marks SHF, Vasoepididymostomy : end to side longitudinal multilaytier intususception. In. Marks SHF, Vasectomy reversal: Manual of vasovasostomy and Vasoepididymostomy. New York, Springer, 2019: 101-127.

[41] Shin D, Lipshultz L, Goldstein M, Barme GA, Fuchs EF, Nagler HM, et al. Herniorrhaphy with polypropylene mesh causing inguinal vassal obstruction, a presentable cause of obstructive azoospermia. Ann Surg 2005; 241: 553-8.

[42] Goldstein M. Surgery for male infertility. Philadelphia, Saunders, 1995.

[43] Goldstein M, Li PS, Mathews GJ. Microsurgical vasovasostomy: the microdot technique of precision suture placement. J Urol 1998; 159: 188-90.

[44] Kolettis PN, Burns JR, Nangia AK, Sandlow JI. Outcomes for vasovasostomy performed when only sperm parts are present in the vassal fluid. J Androl 2006; 27: 565-7.

[45] Scovell JM, Mata DA, Ramasamy R, Herrel LA, Hsiao W, Lipshultz LI, Association between the presence of sperms in the vassal fluid during Vasectomy reversal and postoperative patency: a systematic review and meta-analysis. Urology 2015; 194: 156-9.

[46] Ostrowski KA, Polachwich AS, Conlin MJ, Hedges JC, Fucks EF. Impact on pregnancy of gross and microscopic vasal fluid during Vasectomy reversal. J Urol2015; 194: 156-9.

[47] Anger JT, Goldstein M. Intravasal "toothpaste" in men with obstructive azoospermia is derived from vassal epithelium not sperm. J Urol 2004; 172: 634-6.

[48] Hopps CV, Goldstein M, Schlegel PN. The diagnosis and treatment of the azoospermic patient in the age of intracytoplasmic injection. Urol Clin North Am 2002; 29: 895-911.

[49] Hopps CV, Goldstein M. Outcomes for vasovasostomy with bilateral intravasal azoospermia. J Androl 2003; 24: 25-6.

[50] Hayden RP, Li PS, Goldstein M. Microsurgical Vasectomy reversal: contemporary techniques, intraoperative decision making, and surgical training for the next generation. Fertil Steril 2019: 111: 444-53

[51] Page ST. Physiological roles and regulation of intratesticular sex steroids. Curr Opin Endocrinol Diabetes Obes2011; 18: 217-23.

[52] Gu Y, Liang X, Wu W, Liu M, Song S, Cheng L, et al. Multicentre contraceptive efficacy trial of injectable testosterone undecanoate in Chinese men. J Clin Endocrinol Metab 2009; 94: 1910-5.

[53] Coward RM, Mata DA, Smith RP, Kovac JR, Lipshultz LI. Vasectomy reversal outcomes in men previously on testosterone supplementation therapy. Urology 2014; 84: 1335-40.

[54] McBride JA, Coward RM. Recovery of spermatogenesis following testosterone replacement therapy or anabolic steroids.. Asian J Androl 2016; 18: 373-80.

[55] Shynkin YR, Li PS, Magid ML, Carlson D, Chen EC, Goldstein M. Comparison of absorbable and nonabsorbable sutures for microsurgical vasovasostomy in rats. Urology 1999; 53: 1235-8.

[56] Korkes F, Castro O, Neto N. Crossover transseptal vasovasostomy: alternative for very selected cases of iatrogenic injury to vas deferens. IBJU: Challenging Clinical Cases 2019; 45(2): 392-395.

[57] Liang ZY, Zhang FB, Li LJ, Li JP, Wu JG, Chen C. Clinical application ofcross microsurgical vasovasostomy in scrotum for atypical obstructive azoospermia. I J Zheijiang Univ Sci B (Biomed and Biotechnol) 2019; 20(3): 282-286.

[58] Chawla A, O’Brien J, Lisi M, Zini A, Jarvi K. Should all urologists performing Vasectomy reversal be able to perform Vasoepididymostomies if required?J Urol 2004; 172: 1048-50.

[59] Chan PT, Li PS, Goldstein M. Microsurgical Vasoepididymostomy: A prospective randomized 
An Updates on Techniques of Microsurgical Vasectomy Reversal- A Mini Review

study of 3 intussusception techniques in rats. J Urol 2003; 169: 1924-9.

[60] Zhao L, Deng CH, Sun XZ, Chen Y, Wang WW, Zeng LY, et al. A modified single-armed technique for Microsurgical Vasoepididymostomy. Asian J Androl 2013; 15: 79-82.

[61] Zhang Y, Wu X, Yang XJ, Zhang H, Zhang B. Vasal vessels preserving Microsurgical Vasoepididymostomy in cases of previous varicocoelectomy: a case report and literature review. Asian J Androl 2016; 18: 154-6.

[62] Kuang W, Shin PR, Matin S, Thomas AJr. Initial evaluation of robotic technology for Microsurgical vasovasostomy. J Urol 2004; 171: 300-3.

[63] Schiff J, Li PS, Goldstein M. Robotic Microsurgical vasovasostomy and Vasoepididymostomy: a prospective randomized study in a rat model. J Urol 2004; 171: 1720-5.

[64] Kavoussy PK. Validation of robot -assisted Vasectomy reversal. Asian J Androl 2015; 17: 245-7.

[65] Trost L, Parekatti S, Wang J, Hellstrom WJ, Intracorporeal robot -assisted Microsurgical vasovasostomy for the treatment of bilateral vassal obstruction occurring following bilateral inguinal hernia repairs with mesh placement. J Urol 2014; 191: 1120-5.

[66] Medot M, Nelissen X, Heymans O, Adant JP, Fissette J. Video-microsurgery : a new tool in microsurgery. Br J Plast Surg 1999; 52: 92-6.

[67] Liu J, Chen B, Ni Y, Zhan Y, Gao H. Application of three-dimensionals microsurgical video system for a rat femoral vessel anastamosis. Chin Med J (Eng) 2014; 127: 348-52.
[68] Majzoub A, Tadros NN, Polachwich AS, Sharma R, Agarwal A, Sabanegh E Jr. Vasectomy reversal semen analysis: new reference rates predict pregnancy. Fertil Steril 2017; 107: 911-5.

[69] World Health Organization. In WHO laboratory manual for the examination and processing of semen. $5^{\text {th }}$ ed. Geneva: WHO 2010.

[70] Abboudi H, Khan MS, Guru KA, Froghi S, De Win G, Van Poppel H, etal. Learning curves for urological procedures: a systematic review and meta-analysis. BJU Int 2014; 114: 617-29.

[71] Wood S, Montazeri N, Sajjad Y, Troup S, Kingsland CR, Lewis-Jones DI. Current practice in the management of Vasectomy reversal and unobstructive azoospermia in Merseyside and North Wales: a questionnaire -based survey. BJU Int 2003; 91: 839-44.

[72] Nagler HM, Jung H. Factors predicting successful microsurgical Vasectomy reversal. Urol Clin North Am 2009; 36: 383-90.

[73] Najjari BP, Li PS, Ramasamy R, Katz M, Sheth S, Robinson $\mathrm{B}$, et al. Microsurgical rat varicocoele model. J Urol 2014; 191: 548-53.

[74] Grober ED, Hamstra SJ, Wanzel KR, Reznick RK, Matsumoto ED, Sidhu RS, et al. The educational impact of bench model fidelityon the acquisition of technical skill: the use of clinically relevant outcome measures. Ann Surg 2004; 240: 374-81.

[75] Kavoussi PK, Harlan C, Kavoussi KM, Kavoussi SK. Robot assisted microsurgical vasovasostomy: the learning curve for a pure microsurgeon. J Robotic Surgery 2018; https: //doi10. 1007/s11701-080888-0.

Citation: Dr. Kulvinder Kochar Kaur, Dr. Gautam Allahbadia, Dr. Mandeep Singh. An Updates on Techniques of Microsurgical Vasectomy Reversal- A Mini Review. Archives of Urology. 2019; 2(2): 9-23.

Copyright: (C) 2019 Dr. Kulvinder Kochar Kaur, Dr. Gautam Allahbadia, Dr. Mandeep Singh. This is an open access article distributed under the Creative Commons Attribution License, which permits unrestricted use, distribution, and reproduction in any medium, provided the original work is properly cited. 